This item was submitted to Loughborough's Research Repository by the author.

Items in Figshare are protected by copyright, with all rights reserved, unless otherwise indicated.

\title{
Individual variation in hunger, energy intake and ghrelin responses to acute exercise
}

\section{PLEASE CITE THE PUBLISHED VERSION}

https://doi.org/10.1249/MSS.0000000000001220

\section{PUBLISHER}

(C) American College of Sports Medicine (ACSM)

\section{VERSION}

AM (Accepted Manuscript)

\section{PUBLISHER STATEMENT}

This work is made available according to the conditions of the Creative Commons Attribution-NonCommercialNoDerivatives 4.0 International (CC BY-NC-ND 4.0) licence. Full details of this licence are available at: https://creativecommons.org/licenses/by-nc-nd/4.0/

\section{LICENCE}

CC BY-NC-ND 4.0

\section{REPOSITORY RECORD}

King, James, Kevin Deighton, David R. Broom, Lucy K. Wasse, Jessica Douglas, Stephen F. Burns, Philip Cordery, et al.. 2017. "Individual Variation in Hunger, Energy Intake and Ghrelin Responses to Acute Exercise”. Loughborough University. https://hdl.handle.net/2134/24093. 
1 Individual variation in hunger, energy intake and ghrelin responses to acute exercise

2 King $\mathrm{JA}^{1,2}$, Deighton $\mathrm{K}^{3}$, Broom $\mathrm{DR}^{4}$, Wasse $\mathrm{LK}^{5}$, Douglas $\mathrm{JA}^{1,2}$, Burns $\mathrm{SF}^{6}$, Cordery $\mathrm{PA}^{1,2}$,

3 Petherick $\mathrm{ES}^{1,2}$, Batterham $\mathrm{RL}^{7}$, Goltz $\mathrm{FR}^{1,2}$, Thackray $\mathrm{AE}^{1,2}$, Yates $\mathrm{T}^{2,8}$, Stensel $\mathrm{DJ}^{1,2}$.

4

$5{ }^{1}$ School of Sport, Exercise and Health Sciences, Loughborough University, United Kingdom

$6 \quad$ 2NIHR Leicester-Loughborough, Diet, Lifestyle \& Physical Activity Biomedical Research

7 Unit

$8 \quad{ }^{3}$ Institute for Sport, Physical Activity and Leisure, Leeds Beckett University, United

9 Kingdom

$10{ }^{4}$ Academy of Sport and Physical Activity, Sheffield Hallam University, United Kingdom

$11{ }^{5}$ Faculty of Medical and Human Sciences, University of Manchester, United Kingdom

$12{ }^{6}$ Department of Physical Education \& Sport Science, National Institute of Education,

13 Nanyang Technological University, Singapore

$14{ }^{7}$ Department of Medicine, University College London, United Kingdom

${ }^{8}$ College of Medicine, Biological Sciences and Psychology, University of Leicester, United

16 Kingdom

\section{Correspondence}

19 Dr James King

Lecturer in Exercise Physiology

21 School of Sport, Exercise and Health Sciences

22 Loughborough University

23 Leicestershire

24 United Kingdom 
26 Phone: +44(0)1509 228457

27 E-mail: J.A.King@lboro.ac.uk

28

29 Abstract length : 275 words

30 Manuscript length: 6558 words

31

32

33 
Purpose: To characterise the immediate and extended impact of acute exercise on hunger, energy intake and circulating acylated ghrelin concentrations using a large dataset of homogenous experimental trials; and to describe the variation in responses between individuals. Methods: Data from 17 of our group's experimental crossover trials were aggregated yielding a total sample of 192 young, healthy, males. In these studies, single bouts of moderate to high-intensity aerobic exercise (69 $\pm 5 \% \mathrm{VO}_{2}$ peak; mean \pm SD) were completed with detailed participant assessments occurring during and for several hours postexercise. Mean hunger ratings were determined during $(n=178)$ and after $(n=118)$ exercise from visual analogue scales completed at 30 min intervals whilst ad libitum energy intake was measured within the first hour after exercise $(n=60)$ and at multiple meals $(n=128)$ during the remainder of trials. Venous concentrations of acylated ghrelin were determined at strategic time points during $(n=118)$ and after $(n=89)$ exercise. Results: At group-level, exercise transiently suppressed hunger $(P<0.010$; Cohen's $d=0.77)$ but did not affect energy intake. Acylated ghrelin was suppressed during exercise $(P<0.001$; Cohen's $d=$ 0.10 ) and remained significantly lower than control (no exercise) afterwards $(P<0.024$; Cohen's $d=0.61)$. Between participants, there were notable differences in responses however a large proportion of this spread lay within the boundaries of normal variation associated with biological and technical assessment error. Conclusion: In young men, acute exercise suppresses hunger and circulating acylated ghrelin concentrations with notable diversity between individuals. Care must be taken to distinguish true inter-individual variation from random differences within normal limits. 
The interaction between exercise, appetite and food intake has received widespread scientific attention within recent years given the direct relevance for energy balance and weight control (4). Emergent from this body of research is a consensus that single bouts of moderate- to high-intensity exercise transiently suppress appetite but have no influence on ad libitum energy intake (10,33). Energy homeostasis therefore seems insensitive to acute energy deficits imposed by exercise; with more prolonged or repeated perturbations necessary to induce partial compensatory responses $(36,39)$. In association with this line of research has been a related interest in seeking to understand the mechanisms underpinning appetite control and perturbations in energy balance resulting from exercise and dietary interventions. Notably, the responses of several gut peptides to exercise (acylated ghrelin, peptide YY $Y_{3-36}$, glucagon-like-peptide-1, cholecystokinin) have been scrutinised as possible modulators of appetite and food intake (34). The most consistent finding from these investigations is that exercise transiently alters the circulating concentrations of these hormones in directions associated with suppressed appetite; however, circulating concentrations are typically not different from control at 30 to 60 min post-exercise (10).

With a growing emphasis within biomedical science on 'precision medicine' (2) recent research has sought to characterise the individual variability in appetite and energy intake responses to exercise $(13,18,20,27)$. The primary question addressed within these studies is whether some individuals are more or less likely to compensate for energy expended during exercise by increasing post-exercise energy intake. The implication of this inquiry is that exercise may be less useful for weight management in 'compensators' compared with 'noncompensators'. Unfortunately, to date, the studies which have examined this issue are limited 
variation (technical error and biological variation) (1). Additional research is therefore needed to provide greater insight within this area of research.

Over the last 15 years our research group has conducted many experimental exercise interventions examining the effects of acute exercise on appetite, ad libitum energy intake and appetite-regulatory hormones. Given the uniqueness of acylated ghrelin as the only circulating hormone known to stimulate appetite and promote positive energy balance $(9,40)$, our research has maintained a central focus on the interaction between exercise, appetite, ad libitum energy intake and acylated ghrelin. Usefully, the experimental designs (randomised cross-over trials with exercise and control trials), participants (lean, young, healthy, males) and exercise protocols (aerobic moderate- to high-intensity exercise) utilised within these studies have been remarkably similar. This similarity permits the aggregation of data which provides enhanced power to investigate experimental intervention effects and to interrogate associations between key variables. Uniquely, in this context, this large dataset also provides a novel opportunity to comprehensively explore the variability in appetite and ad libitum energy intake responses to exercise between individuals.

The primary aims of this study were two-fold. Firstly, using our large, pooled dataset of experimental trials, we sought to characterise the immediate (during and shortly after exercise) and extended (several hours post-exercise) impact of acute exercise on perceived hunger, ad libitum energy intake and circulating concentrations of acylated ghrelin. Secondly, with precise consideration of the day-to-day biological and technical error inherent within outcome measurements, we sought to determine the individual variation in hunger, ad libitum energy intake and circulating acylated ghrelin responses, both during and in the hours after a single bout of exercise. To achieve this second aim we have collected new data to determine 
the day-to-day variation (with no intervention) in hunger, circulating acylated ghrelin and energy intake (during ad libitum feeding) in young, healthy males. The findings reported in this manuscript provide novel insights concerning the interaction between exercise, appetite control and energy homeostasis.

\section{METHODS}

\section{Research studies and participants}

The data described in this manuscript were derived from 17 studies (16 published in peer reviewed scientific journals; one currently in press) which were conducted between 2004 and 2014 in the exercise physiology laboratory led by Professor David Stensel at Loughborough University, UK. All included studies received ethical approval from the institutional ethical advisory board and written informed consent was obtained from all participants before any trial procedures commenced. Each trial included within this pooled analysis was an acute randomised-crossover trial with participants having completed paired exercise (see detail below) and control (resting within the laboratory) trials. The key features of each study in this pooled investigation are described in tables within the accompanying Supplementary Digital Content $(1-8)$. In all of the studies the participants $(n=192$ in total) were young ((mean \pm SD) $22.3 \pm 2.7$ years), lean (BMI $23.4 \pm 2.2 \mathrm{~kg} / \mathrm{m}^{2}$ ), recreationally active ( $\dot{\mathrm{VO}}_{2}$ peak $(n=178)$ $57.8 \pm 8.2 \mathrm{~mL} / \mathrm{kg} / \mathrm{min}$ ) males who were metabolically healthy. All of the participants were weight stable ( $<2.5 \mathrm{~kg}$ change in body weight) for at least three months before experimental trials.

\section{Exercise protocol characteristics}

The exercise stimuli imposed within the studies included in this pooled analysis were homogenous; in all instances being characterised as a single bout of moderate- to high- 
intensity aerobic exercise. In all trials, exercise was conducted within a controlled laboratory setting with participants exercising under the direct supervision of study experimenters. In all except one study (which involved an acute bout of swimming), the mode of exercise completed was treadmill running or ergometer cycling with indirect calorimetry (Douglas bags) used to monitor exercise intensity and determine energy expenditure and substrate oxidation (15). Across exercise trials the intensity of exercise ranged from 56 to 83 percent of $\dot{\mathrm{V}} \mathrm{O}_{2}$ peak with a mean intensity of $69 \pm 5 \%$. The duration of each acute exercise bout ranged from 30 to 90 min (30 min, two studies; 60 min, 11 studies; 90 min, four studies).

\section{Anthropometry and standardisation}

Body mass and stature were determined using standard techniques with participants wearing light clothing. Body composition (fat mass and fat-free mass) was determined using skin-fold measurements (triceps, bicep, subscapular, suprailiac) and the published equations of Durnin and Womersley (12) and Siri (35). Participants' age, stature and body mass was used to estimate resting metabolic rate as described by Mifflin et al. (31). Participants refrained from consuming alcohol, caffeine and participating in structured exercise for 24-48 h before main experimental trials and during this period dietary intake was standardised using weighed food records. Participants' last meal was consumed before study days on the prior evening (no later than 22:00) and all main trials commenced the following morning after an overnight fast. Participants maintained their habitual diet between trials in all experiments.

\section{Hunger analyses}

The primary analyses of interest in this study relating to hunger were: 1) individual variation in fasting hunger ( $n=192$ ); 2) the immediate (during exercise, $n=178$ ) and prolonged (up to 8 h post-exercise, $n=118$ ) effects of exercise on perceived hunger. In each of the studies 
included within these analyses participants reported their perceived hunger at intervals of 30 min using pen and paper based $100 \mathrm{~mm}$ visual analogue scales (14). The impact of exercise on hunger was assessed by comparing mean hunger ratings calculated during and after exercise with paired values calculated on each participant's control trial. In the post-exercise hunger analysis mean hunger scores were calculated from data available until the end of trials or until the occurrence of a buffet meal (when standardised appetite scores were no longer comparable). The reproducibility of fasting perceived hunger was determined from baseline hunger ratings at the start of paired exercise and control trials. Individual variation in hunger responses during and after exercise were calculated by subtracting mean hunger ratings 30 min after exercise was excluded to eliminate any latent impact of the exercise bout.

In order to examine the individual variation in hunger responses during and after exercise we compared each participant's response with our new data ( $n=15$ young, healthy males) regarding the variation in hunger ratings across one hour (most common duration of exercise in the present analyses) (1 h: $\pm 30 \mathrm{~mm} ; 17.2 \%)$ and over an extended duration (2.5 h: \pm 20 $\mathrm{mm} ; 13.8 \%)$ with no intervention.

\section{Energy intake analyses}

179 The primary analyses of interest relating to exercise and ad libitum energy intake were: 1) the impact of acute exercise on energy intake at the first meal consumed shortly after exercise (within $60 \mathrm{~min})(n=60) ; 2)$ the impact of acute exercise on energy intake across several hours post-exercise (range 5 - 9 h) $(n=128)$. In each of the studies included within these analyses, ad libitum energy intake was determined from buffet-style meals whereby 
participants had access to a range of foods for a discrete period of time (30 mins) which was identical on paired exercise and control trials. In all trials, participants were instructed to eat until 'comfortably full and satisfied' and that additional food was available if desired. All meals were consumed in isolation so that social factors did not influence eating behaviour. Variation in energy intake responses to exercise was determined by subtracting each participant's energy intake during the control trial from their intake during paired exercise trials. Within the analyses examining the delayed effects of exercise on energy intake, data was included only if participants had remained in the laboratory during the entire period of exercise (which occurred in three studies identified within this paper). variation’ in energy intake responses in the present investigation. observation. Additionally, data was only assessed from meals consumed on the same day as exercise i.e. data was not included from energy intake assessments conducted on the day after

Because the natural day-to-day variability in energy intake is highly dependent on the participants studied and the format of ad libitum meal provision (i.e. homogenous meal versus buffet meal and types of foods available at laboratory meals), we conducted a new study to characterise the variation in ad libitum energy intake across two meals (breakfast and lunch) when using a buffet meal (24) (Appendix 1) and participant cohort ( $n=18$; healthy, lean males) identical to that utilised within the studies described in the present manuscript. In this setting we found that the co-efficient of repeatability and intra-subject variation at breakfast was $\pm 1937 \mathrm{~kJ}$ and $18.9 \%$. Furthermore, when energy intake at breakfast was combined with a buffet lunch, together, the corresponding repeatability values were $2138 \mathrm{~kJ}$ and $8.9 \%$. These boundaries of variation were used to determine the boundaries of 'true 


\section{Acylated ghrelin analyses}

210 The primary analyses of interest relating to acylated ghrelin were: 1 ) the immediate (during exercise, $n=118$ ) and prolonged (up to $8 \mathrm{~h}$ post-exercise; $n=89$ ) effects of acute exercise on

212 circulating acylated ghrelin concentrations; 2) day-to-day variation in fasting circulating acylated ghrelin concentrations $(n=138)$. In each of the studies included within these analyses circulating concentrations of acylated ghrelin were determined from venous blood samples taken by venepuncture (fasting measurement in one study) or cannulas (16 studies) positioned in antecubital veins. Across all studies, plasma acylated ghrelin concentrations were determined using the same enzyme-linked immune-sorbent assay (SPI-BIO, Montigney le Brettoneux, France) which has demonstrated good intra-assay (typically 6-8\%) variation in our laboratory. Importantly, identical sampling pre- and post-treatment was performed across all studies as detailed previously (6). Variation in circulating acylated ghrelin responses to exercise was determined by subtracting the plasma acylated ghrelin AUC during the period of interest within the control trial (exercise period and post-exercise period) from the corresponding period during the exercise trial. These data were then expressed as a percentage difference with positive values indicating an increase in circulating acylated ghrelin in response to exercise (and vice-versa). Acylated ghrelin data was expressed as percentage difference, rather than absolute values (as per our hunger and energy intake data), due to variation in absolute acylated ghrelin values obtained across our data (most likely related to antibody variation with ELISA kits over time). To determine the day-to-day variability in circulating acylated ghrelin concentrations over an extended period, we collected new data whereby circulating acylated ghrelin concentrations were determined from six samples over a $2.5 \mathrm{~h}$ period on two separate days with no intervention ( $n=15$ healthy, young males). With diet and physical activity standardised in the prior $24 \mathrm{~h}$, across a period of $1 \mathrm{~h}$ (the median exercise duration in the present analysis), the co-efficient of repeatability 
and intra-subject variation for circulating acylated ghrelin was $\pm 46 \mathrm{pg} / \mathrm{mL}$ and $17.2 \%$, respectively. Over a longer period of $2.5 \mathrm{~h}$ the corresponding values were $\pm 38 \mathrm{pg} / \mathrm{mL} / \mathrm{h}$ and $14.4 \%$.

\section{Statistical analyses}

Data was analysed using the Statistical Package for the Social Sciences (SPSS) software version 22.0 (IBM SPSS, Inc., Chicago, IL). Area under the curve (AUC) was calculated for plasma acylated ghrelin using the trapezoidal method. Repeated measures analysis of covariance (ANCOVA) were used to assess differences in hunger (fasting and mean values), energy intake and circulating acylated ghrelin (fasting and AUC) between paired control and exercise trials. Study was included as a covariate for all analyses whilst additional covariates were added if they correlated significantly with dependent variables. In effect, age and fat mass were included as additional covariates in the fasting hunger analyses whilst fat mass was included as a covariate in the post-exercise hunger analyses. Variation in fasting hunger ratings and circulating acylated ghrelin concentrations were expressed as the co-efficient of intra-subject variation $\left(\mathrm{CV}_{\text {intra }}=\mathrm{SDd} /(\mathrm{m} \sqrt{2})\right)$ and co-efficient of repeatability $(\mathrm{CR}=2 \mathrm{x} \mathrm{SD})$ as described by Horner et al (21). The Person product-moment correlation co-efficient was used to examine relationships between key variables with the correlations interpreted as small (0.1), medium (0.3), and large (0.5) (8). Within the correlation analyses exact participant numbers are stated in parenthesis when this deviates from the number included within the main outcome analysis. Effect sizes were calculated to determine the magnitude of statistical effects using Cohen's $d$ which adopts the following values to represent small (0.2), medium (0.5) and large (0.8) effects (8). All data are presented as mean \pm standard deviation. Statistical significance was identified if $P<0.05$. 


\section{RESULTS}

\section{Hunger responses}

261 Data describing paired fasting hunger scores at the beginning of an exercise and control trial was available for 192 participants (see table; Supplementary Digital Content 1). There was no significant difference in fasting hunger scores between trials (exercise $59 \pm 23 \mathrm{~mm}$; control $56 \pm 24 \mathrm{~mm} ; P=0.929 ; d=0.13)$. The intra-subject variation in fasting hunger between paired exercise and control trials was $38 \%$ with a co-efficient of repeatability of $\pm 44 \mathrm{~mm}$. Fasting hunger was strongly correlated between each participant's main trials $(r=0.557, P<$ 0.001). Mean fasting hunger scores were positively associated with fat-free mass ( $n=165 ; r$ $=0.213 ; P=0.006)$ and age $(r=0.143 ; P=0.048)$ and inversely related to fat mass $(n=165$; $r=-0.213 ; P=0.006)$. Mean fasting hunger was not related to weight $(r=-0.032 ; P=0.662)$, BMI $(r=-0.045 ; P=0.537), \dot{V}_{2}$ peak $(n=178 ; r=-0.057 ; P=0.450)$ or estimated resting metabolic rate $(r=-0.039 ; P=0.591)$.

The tables in Supplementary Digital Content 2 and 3 identify the specific studies, along with their associated characteristics, which were pooled to obtain data regarding hunger responses during $(n=178)$ and after $(n=118)$ exercise. Mean hunger ratings during exercise were significantly lower compared with paired hunger ratings during control trials (exercise $41 \pm 26$ $\mathrm{mm}$; control $61 \pm 22 \mathrm{~mm} ; P=0.010 ; d=0.77)$. Figure 1 a shows each participant's net individual hunger response during exercise (difference between exercise and control) and demonstrates the wide range of responses observed $(-94$ to $+73 \mathrm{~mm})$. Notably, $79 \%(n=$ 140) of participants demonstrated suppressed hunger during exercise whilst $19 \%(n=34)$ documented an increase ( $2 \%$ showed no difference between control and exercise trials). Importantly, however, when considering the natural variation in hunger assessment with no intervention ( $\pm 30 \mathrm{~mm}$ over one hour) it can be seen that $37 \%(n=65)$ of participants’ hunger 
was suppressed to an extent greater than the boundaries of normal variation whilst $3 \%(n=5)$ demonstrated an increase. The remaining 60\% $(n=108)$ lay within this boundary. Further scrutiny of these data revealed a weak inverse relationship between percent carbohydrate oxidation during exercise and mean hunger $(n=152 ; r=-0.177 ; P=0.030)$. There were no relationships between mean hunger during exercise and fat oxidation $(n=152 ; r=0.079 ; P=$ 0.332), exercise intensity ( $n=162 ; r=-0.100 ; P=0.204)$, energy expenditure $(n=162 ; r=-$ 0.105; $P=0.182)$ or $\dot{\mathrm{V}} \mathrm{O}_{2}$ peak $(n=164 ; r=-0.088 ; P=0.260)$.

\section{Insert figure 1 here}

Hunger responses after exercise were analysed using data collected up until the end of trials, or until the provision of an ad libitum meal (range 3-8 h post-exercise). There was no significant difference in mean hunger ratings after exercise between the paired exercise $(44 \pm 17 \mathrm{~mm})$ and control trials $(44 \pm 18 \mathrm{~mm})(P=0.142 ; d=0.01)$. Figure $1 \mathrm{~b}$ shows the aggregate of each participant's post-exercise mean hunger responses which varied widely $(-52$ to $+30 \mathrm{~mm})$. Fifty percent $(n=59)$ of participants reported lower mean post-exercise hunger whilst 47\% $(n=56)$ demonstrated higher mean post-exercise hunger (3\% reported no difference between trials). Importantly, when normal variation is considered, $90 \%(n=106)$ of participants' responses lay within the boundaries of normal variation with $4 \%(n=5)$ demonstrating higher mean hunger after exercise and 6\% $(n=7)$ reporting lower. Within these studies, we detected a small significant correlation between post-exercise hunger and fat oxidation during exercise ( $n=106 ; r=-0.247 ; P=0.011$ ). No relationships were found between mean post-exercise hunger and carbohydrate oxidation $(n=106 ; r=-0.011 ; P=$ 0.911), age ( $n=118 ; r=-0.062 ; P=0.504)$, BMI $(n=118 ; r=-0.055 ; P=0.552)$, weight $(n$ $=118 ; r=0.032 ; P=0.730)$, fat-free mass $(n=107 ; r=-0.081 ; P=0.404)$, fat mass $(n=$ 
$107 ; r=0.082 ; P=0.402)$, energy expenditure $(n=116 ; r=0.162 ; P=0.082)$ or exercise intensity $(n=116 ; r=0.108 ; P=0.250)$.

\section{Energy intake responses}

Data was pooled from five of our previous research studies $(n=60)$ to explore the diversity of ad libitum energy intake responses at one meal provided within 60 min after a single bout of moderate- to high-intensity aerobic exercise. The table within Supplementary Digital Content 4 describes the characteristics of the individual studies included. As a group, there was no significant difference in energy intake between paired exercise and control trials (exercise $5899 \pm 1778 \mathrm{~kJ}$; control $5770 \pm 1966 \mathrm{~kJ})(P=0.977 ; d=0.10)$ with energy intake between trials showing a strong positive correlation $(P<0.001 ; r=0.688)$. Figure 2a shows that on a crude individual basis there was a range of responses observed (-5005 to $+4389 \mathrm{~kJ})$ with 55\% ( $n=33)$ of participants consuming more and 45\% $(n=27)$ consuming less after exercise. Importantly though, when these data are compared against the natural variation in ad libitum energy intake at one meal with no intervention ( $\pm 1937 \mathrm{~kJ} ; 18.9 \%)$ it is apparent that $85 \%(n=51)$ of participants exhibited responses within this boundary of normal variation. Seven percent of participants $(n=4)$ documented reduced post-exercise energy intake beyond this boundary whilst $8 \%(n=5)$ showed an increase above this boundary.

\section{Insert figure 2 here}

In this cohort there was no relationship between post-exercise energy intake and prior energy expenditure ( $r=0.054 ; P=0.720)$, exercise intensity $(r=0.029 ; P=0.850)$, carbohydrate $(r$ $=0.113 ; P=0.454)$ or fat oxidation $(r=-0.049 ; P=0.746)(n=46)$. Hunger ratings 
immediately before the first post-exercise meals were lower after exercise, likely reflecting a delayed appetite suppressive effect (exercise $59 \pm 28 \mathrm{~mm}$; control $64 \pm 23 \mathrm{~mm} ; P=0.006$; $d$ $=0.36$ ). Despite this, pre-meal hunger did not correlate with subsequent energy intake at the first post-exercise meal in the control $(r=0.158 ; P=0.229)$ or exercise trials $(r=-0.019 ; P=$ 0.886) $(n=60)$.

To examine the influence of acute exercise on food intake over the course of entire laboratory trial days, including multiple ad libitum meals in some instances, data from a further six studies were pooled ( $n=128$ ) (see table; Supplementary Digital Content 5). Three of the 11 studies provided data from two ad libitum meals, the remainder utilised one meal (which was provided $>1 \mathrm{~h}$ post-exercise). As a group, there was no significant difference in energy intake between paired exercise and control trials (exercise $9694 \pm 5468 \mathrm{~kJ}$; control $9498 \pm$ $5435 \mathrm{~kJ} ; P=0.481 ; d=0.11$ ) with responses between trials showing a strong positive correlation $(P<0.001 ; r=0.949)$. Figure $2 \mathrm{~b}$ shows that on a crude individual basis there was a range of responses observed; 59\% $(n=75)$ of participants consumed more and $41 \%(n=$ 53) consumed less after exercise. Importantly though, when these data are compared against the natural variation in ad libitum energy intake from multiple meals with no intervention $( \pm$ $2138 \mathrm{~kJ} ; 8.9 \%)$, it is apparent that $81 \%(n=105)$ of participants exhibited responses within this boundary of normal variation (Figure 2b). Nine percent $(n=11)$ of participants documented reduced post-exercise energy intake beyond this boundary whilst $10 \%(n=12)$ showed an increase. Across the control $(r=0.592)$ and exercise trials $(r=0.623)$ ad libitum energy intake was associated with hunger ratings (both $P<0.001$ ) determined after exercise (or the equivalent time period on the control trial). 


\section{Acylated ghrelin responses}

360 Data describing paired fasting acylated ghrelin plasma concentrations was available for 141 participants (see table; Supplementary Digital Content 6). Two outliers were identified and removed from these analyses because the difference between paired samples was 4.5 and 10.5 fold greater than the standard deviation of differences between paired samples for the cohort $( \pm 31 \mathrm{pg} / \mathrm{mL})$. One additional outlier was removed because their mean fasting plasma acylated ghrelin values were 7.7 times greater than the group mean $(949 \mathrm{pg} / \mathrm{mL}$ vs. 123 $\mathrm{pg} / \mathrm{mL})$. With these outliers removed $(n=138)$, fasting acylated ghrelin plasma concentrations did not differ between the control $(125 \pm 109 \mathrm{pg} / \mathrm{mL})$ and exercise $(121 \pm 100$ $\mathrm{pg} / \mathrm{mL})$ trials $(P=0.638, d=0.12)$. The coefficient of repeatability and intra-subject variation between samples was $\pm 63 \mathrm{pg} / \mathrm{mL}$ and $19.2 \%$, respectively. There were no significant correlations between mean fasting acylated ghrelin and hunger $(r=-0.004 ; P=0.959)$, BMI $(r=-0.093 ; P=0.275)$, weight $(r=-0.091 ; P=0.288)$, age $(r=-0.015 ; P=0.860)$, estimated resting metabolic rate $(r=-0.073 ; P=0.392)$, fat-free mass $(n=114 ; r=0.092 ; P=0.331)$ or fat mass $(n=114 ; r=-0.092 ; P=0.331)$.

Acylated ghrelin responses during exercise were examined using data derived from 12 studies ( $n=118$, see table in Supplementary Digital Content 7). In eight studies the duration of exercise was 60 min (80 participants); in three studies it was 90 min (30 participants) and in one study it was 30 min (eight participants). As a group, the circulating acylated ghrelin AUC was 24\% lower during exercise (99 $\pm 94 \mathrm{pg} / \mathrm{mL} / \mathrm{hour})$ compared with control (131 \pm 106 $\mathrm{pg} / \mathrm{mL} /$ hour $)(P<0.001 ; d=1.0)$. Figure 3a shows the wide variation in acylated ghrelin responses to exercise with $89 \%(n=105)$ of participants exhibiting lower values on their exercise trial while $11 \%(n=13)$ demonstrated higher values after exercise. Notably, when comparing these responses to the natural variation in acylated ghrelin measurement over this 
period $( \pm 17.2 \%$, obtained from our new data) it can be seen that $27 \%(n=32)$ of participants demonstrate responses which fall within this normal range, with 66\% $(n=78)$ and $7 \%(n=8)$ showing a suppression and increase beyond of this range, respectively. No significant correlations were found between acylated ghrelin concentrations during exercise and exercise intensity $(r=-0.111 ; P=0.251)$ or carbohydrate oxidation $(r=0.122 ; P=0.223)$. Fat oxidation during exercise was positively associated with acylated ghrelin concentrations $(r=$ $0.286 ; P=0.004)$.

The prolonged effects of exercise on circulating acylated ghrelin concentrations were assessed by comparing paired post-exercise acylated ghrelin AUC values across nine studies ( $n=89$, see the table in Supplementary Digital Content 8). Plasma acylated ghrelin concentrations were measured between 3-8 h after exercise. As a group, the post-exercise acylated ghrelin AUC was 16\% lower after exercise (108 $\pm 101 \mathrm{pg} / \mathrm{mL} / \mathrm{hour}$ ) compared to control $(128 \pm 120 \mathrm{pg} / \mathrm{mL} /$ hour $)(P=0.024 ; d=0.61)$. Individually, Figure 3b shows that $74 \%(n=66)$ of participants demonstrated reduced levels of acylated ghrelin whilst $26 \%(n=$ 23) showed an increase after exercise. Notably, again, when comparing these responses with the natural acylated ghrelin sampling variation seen across an extended period $( \pm 14.4 \%)$, $42 \%(n=37)$ of participants' responses were within the boundaries defined by this normal variation whilst $10 \%(n=9)$ and $48 \%(n=43)$ of participants' responses were above and below this range, respectively.

\section{Insert figure 3 here}


410 In this study we have pooled our research group's expansive data archive of acute

411 experimental research trials in an effort to provide novel insights regarding the interaction

412 between exercise and appetite regulation. Specifically, in this paper, the data from 17 of our

413 group's previous studies have been collated to interrogate interactions between exercise, hunger, ad libitum energy intake and acylated ghrelin. Importantly, this large database of tightly controlled experimental trials has enabled us to explore inter-subject variation in response to exercise which is a key consideration in precision medicine and has begun to receive attention in energy balance research $(13,18,20,38)$. Our findings clarify and consolidate several previously reported outcomes yet also provide new insights which have emerged from our unique collection of data.

The hunger outcomes reported here are consistent with previous findings published within and external to our laboratory which have shown that single bouts of moderate- to highintensity aerobic exercise transiently suppress hunger but have little impact in the hours afterwards $(22,23,25,26,29,30,37)$. Specifically, in our pool of 178 individuals, group-level analyses showed that mean hunger perceptions are suppressed by approximately one-third during exercise which represents a medium- to large-sized statistical effect. Interestingly, there was marked variation in hunger responses which ranged from an extensive suppression to hunger stimulation. Importantly though, even when we accounted for the natural day-today variation in hunger assessment that occurs when using visual analogue scales, we saw that just over one-third of the study sample reported suppressed hunger below this boundary of variation whilst only a handful of individuals reported increased hunger above this level. 
therefore it is uncertain whether or not these responses represent true effects or random variation.

It is relevant to note that in our analyses we compared our hunger data to hunger variability estimates derived from a sample of young, healthy males within our laboratory. We purposefully chose to collect this new data so that our comparator values were derived from the same population and under the same circumstances as per the experimental studies included within this manuscript. Our variability estimates showed that mean hunger can vary by $\pm 30 \mathrm{~mm}$ over the course of one hour which was greater than with additional assessments over a longer period of observation $(2.5 \mathrm{~h}: \pm 20 \mathrm{~mm})$. Variability estimates for hunger ratings calculated over extended durations have been published previously by others and which have ranged $\pm 14-24 \mathrm{~mm}(14,16,21,32)$. These values compare favourably with ours over an extended period and support the validity of our comparisons. This new information shows that despite a large amount of variability being apparent in short-term hunger assessments; exercise is associated with a robust suppression of hunger for a large proportion of individuals. Additional work is now needed to examine whether this effect of exercise is reproducible across exposures within individuals and to identify the key moderating factors.

Our analyses of hunger responses in the hours after exercise demonstrated that single bouts of moderate- to high-intensity aerobic exercise have no impact on hunger during the remainder of the day thereafter for the majority of individuals. Again, this outcome is consistent with previous findings and confirms that acute exercise-induced energy deficits do not create an automatic drive to increase hunger (5). Notably, our data showed an even spread of net mean hunger responses post-exercise; however, the vast majority of responses (90\%) lay within

457 reported boundaries of normal variation. Consequently, our data shows that there is little 
definitive variation in post-exercise hunger responses, with only $10 \%$ of individuals demonstrating changes in post-exercise hunger outside of the normal variation boundaries. In future studies it would be interesting to see whether these responses are consistent across additional trials for this sub-set of individuals as opposed to representing random events.

Given the large number of fasting hunger ratings $(n=192)$ obtained at the beginning of the paired control and exercise trials, we examined the variation between repeated assessments. We identified a rather large variation in fasting hunger (38\%, $\pm 44 \mathrm{~mm})$ which is consistent with results from previous studies. Specifically, in a sample of 12 active males, Gonzalez et al (16) reported a $21 \%$ co-efficient of variation whilst in a similar population others have calculated higher estimates (24-30\%) (32). Furthermore, Horner et al (21) reported a higher estimate in a sample of overweight and obese males (35\%). Collectively, these data identify the expected variation in fasting hunger ratings across repeated assessments in young, healthy males and these data have implications for sample size calculations within experimental research trials. Such high co-efficients of variation also support the measurement of hunger perceptions at multiple time-points in response to an intervention rather than single fasted values.

In our fasting hunger data we identified significant, albeit weak, correlations with fat-free mass (positive) and fat mass (inverse). These findings support recent suggestions that fat-free mass is a central driver of daily food intake (4) whilst adipose tissue may exert an inhibitory effect on appetite and food intake in lean individuals (3). Homogeneity in our participants' body composition may explain the lower strength of these associations in our cohort compared with other published data (3). Alternatively, this discrepancy may be attributable to the correlational rather than causal relationships between these variables. 
In our analyses we also examined the impact of acute exercise on ad libitum energy intake at buffet meals consumed within 60 min after exercise as well as at meals consumed over several hours post-exercise. Consistent with previous data collected outside of our laboratory (25, 26, 28, 33), our pooled analysis showed that at group-level, energy intake was unaffected at meals consumed within the first post-exercise hour. This outcome was apparent, despite hunger ratings being significantly lower (8\%) immediately before ad libitum meals following exercise. Indeed, we actually found that $85 \%$ of participants' net energy intake responses (aggregate of control and exercise values) lay within the boundaries of normal day-to-day variation, as determined by our own repeatability experiment which was conducted with a similar population and buffet meal. This is an important finding because it demonstrates that there is actually very little true variation in ad libitum energy intake beyond the summated boundaries of biological variation and technical measurement error. Previously, researchers have attempted to categorise individual participants as 'compensators' or 'non-compensators' with regards to the effect of exercise on energy intake based upon aggregated energy intake responses after paired acute exercise and control trials $(13,20)$. In these previous studies, it can be seen however, that the net impact of exercise on energy intake is actually less than the natural variation in energy intake from an ad libitum meal which has been defined as \pm 1406 $1477 \mathrm{~kJ}(9-12 \%)$ with ad libitum homogenous meals $(17,21)$ and $\pm 1937 \mathrm{~kJ}$ (18.9\%) with ad libitum buffet meals (latter reported in this paper). Moreover, a recent study has elegantly 502 demonstrated that energy intake responses after exercise show a marked degree of 503 inconsistency; collectively meaning that individuals cannot reliably be classified as 504 'compensators' or 'non-compensators' based upon their energy intake responses to acute exercise (38). Consequently, it is likely that in our analyses, the $15 \%$ of participants who reported exercise-induced alterations in energy intake beyond normal variation boundaries may not exhibit this same response if trials were repeated. 
508

509

510

511

512

513

514

515

516

517

518

519

520

521

522

523

524

525

526

527

528

529

530

531

532

In our energy intake analysis it is worth noting that the identified variability estimates for our ad libitum buffet meals were considerably higher $( \pm 1937 \mathrm{~kJ}, 18.9 \%)$ than previously reported when homogenous meals are provided $(17,21)$. This is most likely because a small change in food selection with a buffet meal on one occasion can produce large differences in energy intake across paired eating assessments. The implication of this is that for studies simply concerned with intervention effects on ad libitum energy intake, rather than food selection, a homogenous meal will reduce the variance in energy intake measurement and increase statistical power.

Our analyses are the first to examine the variation in energy intake responses to multiple meals over several hours after exercise. Again, our findings show that exercise had no impact on energy intake across this extended period. Furthermore, the vast majority of variation in responses once more lay within the boundaries of normal variation that we have determined ourselves across two ad libitum buffet meals. Our results therefore confirm previous findings demonstrating little impact of exercise on energy intake over extended periods (28) and highlight the lack of true variability in responses.

In this manuscript we report the test-retest variability in circulating fasting acylated ghrelin concentrations which has been calculated from a large sample of healthy males. We saw no significant difference in fasting acylated ghrelin concentrations between paired trials. This outcome supports the findings of Chandarana et al. (7) who also observed no differences in fasting or postprandial plasma acylated ghrelin concentrations, with or without dietary standardisation. Despite this, in our analyses, we identified a rather large variance in fasting plasma concentrations ( 19\%) even with prior (24 h) dietary and physical activity standardisation. This variance is composed of the technical error associated with the assay 
measurement (typically 6-8\% in our laboratory) and biological variation in ghrelin secretion and clearance. For the participants in these analyses, dietary standardisation relied on individuals accurately maintaining and subsequently following food diaries and it is possible that biological error could be reduced if diet is standardised for a longer period, or if participants are provided with all of their foods during the standardisation phase. Future research should examine these methodological factors as it has direct relevance for appetite and gut hormone assessment in experimental appetite-regulation research.

A recent meta-analysis of 18 datasets showed that acute exercise transiently supresses circulating concentrations of acylated ghrelin with a small (Cohen's $d-0.2)$ effect size (34). Half of the datasets from this analysis were from our laboratory and therefore it is unsurprising that in the present analysis we identified a statistically large exercise-induced suppression of circulating acylated ghrelin during exercise. The larger effect reported in our laboratory compared with others is likely related to the characteristics of studies, particularly the exercise intensity imposed, and also to variation in assays utilised. Importantly, our data shows that circulating levels of acylated ghrelin are suppressed in response to acute exercise in the vast majority of individuals examined. Of primary significance, in two-thirds of these cases the reduction was beyond the boundaries of normal variation which we explicitly defined for the purpose of this report. This finding highlights the consistency in the response to exercise yet poses the question of why such robust changes were not seen in the remainder of the study sample. Furthermore, the significance of this response is not fully understood and may be unrelated to appetite given that acute changes in response to exercise have not been found to be correlated consistently. In addition to this, although there have been many speculations (19), the mechanism(s) responsible for the exercise related perturbation of acylated ghrelin remain unclear. 
In the present analysis we identified a statistically significant reduction in circulating acylated ghrelin over the course of several hours post-exercise. This finding is interesting given that after exercise has not been identified consistently. The substantially larger study sample used in this pooled analysis was therefore necessary to identify this small statistical effect. Interestingly, our data shows that this persistent effect of exercise can be seen robustly in almost half of participants who exhibited suppressed ghrelin levels after exercise that were beyond the calculated range associated with normal variation. Research is now needed to identify the mechanisms producing this effect and to understand its physiological/metabolic significance.

The analyses in this paper have provided a novel insight regarding the interaction between exercise, hunger, ad libitum energy intake and circulating acylated ghrelin. These analyses have been made possible by the integration of over 10 years of experimental appetite research

572 in our laboratory using study protocols with a high degree of similarity. Our findings do however have some limitations which should be recognised. The first important consideration is the generalisability of our data. Because all of our participants were young, healthy men, we do not know whether our findings would generalise to other populations such as women, children, those who are inactive or obese. A second limitation of our data is that our 577 homogenous sample may have inhibited the ability to identify associations between key 578 variables reported in this paper. Thirdly, it is feasible that the energy intake response to exercise may differ between a laboratory controlled environment and an ecologically valid social setting. However, the aim of this study was to understand the physiological effects of exercise on appetite and energy intake responses in a tightly controlled laboratory environment to control against other confounding factors. Finally, it should be recognised 
that the studies included in the present investigation involved acute exercise protocols that commenced either in the fasted state $(n=13)$ or after a breakfast snack $(n=4)$. Although our group have shown previously that appetite and energy intake responses to acute exercise do not differ depending on feeding status (11), there is the possibility that this factor could have interacted differently across the various studies in our pooled analyses.

In conclusion, our large pooled dataset confirms that single bouts of moderate- to highintensity aerobic exercise transiently, yet robustly, supress hunger but have no impact on ad libitum energy intake across meals consumed on the day of exercise in healthy young men. Additionally, our data shows that exercise robustly suppresses circulating concentrations of acylated ghrelin which in this novel analyses was shown to remain suppressed for several hours after exercise. Importantly, our findings underscore the necessity to consider normal day-to-day variation in these outcomes when examining variability in responses between individuals. Most notably, our research shows that in response to acute exercise, there is very little true variation in post-exercise hunger and energy intake.

\section{ACKNOWLEDGEMENTS}

This research was supported by the National Institute for Health Research (NIHR) Diet, Lifestyle and Physical Activity Biomedical Research Unit based at University Hospitals of Leicester and Loughborough University. The views expressed are those of the authors and not necessarily those of the NHS, the NIHR or the Department of Health.

\section{CONFLICT OF INTEREST}

All authors declare that there are no conflicts of interest. The results of the present study do not constitute endorsement by ACSM. 


\section{REFERENCES}

1. Atkinson G, Batterham AM. True or false interindividual differences in the physiological response to an intervention. Exp Physiol. 2015; 100(6): 577-588.

2. Bayer R, Galea S. Public health in the precision medicine era. N Engl J Med. 2015; 373(6): 499-501.

3. Blundell JE, Finlayson G, Gibbons C, Caudwell C, Hopkins M. The biology of appetite control: do resting metabolic rate and fat-free mass drive energy intake? Physiol Behav. 2015; 152(S1): 473-478.

4. Blundell JE, Gibbons C, Caudwell P, Finlayson G, Hopkins M. Appetite control and energy balance: impact of exercise. Obes Rev. 2015; 16(1): 67-76.

5. Blundell JE, Stubbs RJ, Hughes DA, Whybrow S, King NA. Cross talk between physical activity and appetite control: does physical activity stimulate appetite? Proc Nurt Soc. 2003; 62(3): 651-661.

6. Broom DR, Stensel DJ, Bishop NC, Burns SF, Miyashita M. Exercise-induced suppression of acylated ghrelin in humans. J Appl Physiol. 2007;102(6):2165-71.

7. Chandarana K, Drew ME, Emmanuel J, et al. Subject standardization, acclimatisation and sample processing affect gut hormone levels and appetite in humans. Gastroenterology. 2009; 136(7): 2115-2126. 
8. Cohen J. Statistical power analysis for the behavioural sciences. Hillsdale, NJ: Lawrence Erlbaum Associates; 1969. 23 p.

9. Cummings DE. Ghrelin and the short- and long-term regulation of appetite and body weight. Physiol Behav. 2006; 89(1): 71-84.

10. Deighton K, Stensel DJ. Creating an acute energy deficit without stimulating compensatory increases in appetite: is there an optimal exercise protocol? Proc Nutr Soc. 2014; 73(2): 352-358.

11. Deighton K, Zahra JC, Stensel DJ. Appetite, energy intake and resting metabolic responses to 60 min treadmill running performed in a fed versus a postprandial state. Appetite. 2012; 58(3): 946-954.

12. Durnin JVGA, Wormersley J. Body fat assessment from total body density and its estimation from skinfold thickness: measurements on 481 men and women aged from 16 to 72 years. Br J Nutr. 1974; 32(1):77-97.

13. Finlayson G, Bryant E, Blundell JE, King NA. Acute compensatory eating following exercise is associated with implicit hedonic wanting for food. Physiol Behav. 2009; 97(1): 62-67.

14. Flint A, Raben A, Blundell JE, Astrup A. Reproducibility, power and validity of visual analogue scales in assessment of appetite sensations in single test meal studies. Int J Obes. 2000; 24(1):38-48. 
15. Frayn KN. Calculation of substrate oxidation rates in vivo from gaseous exchange. $J$ Appl Physiol. 1983;55(2):628-34.

661

16. Gonzalez JT, Veasey RC, Rumbold PL, Stevenson EJ. Consistency of metabolic responses and appetite sensations under postabsorptive and postprandial conditions. Appetite. 2012; 59(2): 228-233.

665

17. Gregersen NT, Flint A, Bitz C, Blundell JE, Raben A, Astrup A. Reproducibility and power of ad libitum energy intake assessed by repeated single meals. Am J Clin Nutr.

669

18. Hagobian TA, Yamashiro M, Hinkel-Lipsker J, Streder K, Evero N, Hackney T. Effects of acute exercise on appetite hormones and ad libitum food intake in men and women. Appl Physiol Nutr Metab. 2013; 38(1): 66-72.

673 2008; 87(5): 1277-1281.

677

20. Hopkins M, Blundell JE, King NA. Individual variability in compensatory eating 679 680 following acute exercise in overweight and obese women. Br J Sports Med. 2014; 48(20): 1472-1476. 
699

700

21. Horner KM, Byrne NM, King NA. Reproducibility of subjective appetite ratings and ad libitum test meal energy intake in overweight and obese males. Appetite. 2014; 81: 116-122.

22. Howe SM, Hand TM, Larson-Meyer DE, Austin KJ, Alexander BM, Manore MM. No effect of exercise intensity on appetite in highly trained women. Nutrients. 2016; 8: 233.

23. Kawano H, Mineta M, Miyashita M, et al. Effects of different modes of exercise on appetite and appetite regulating hormones. Appetite. 2013; 66: 26-33.

24. King JA, Wasse LK, Broom DR, Stensel DJ. Influence of brisk walking on appetite, energy intake and plasma acylated ghrelin. Med Sci Sports Exerc. 2010; 42(3): 485492.

25. King NA, Blundell JE. High fat foods overcome the energy expenditure induced by high intensity cycling or running. Eur J Clin Nutr. 1995; 49(2): 114-123.

26. King NA, Burley VJ, Blundell JE. Exercise-induced suppression of appetite: effects on food intake and implications for energy balance. Eur J Clin Nutr. 1994; 48(10): 715-724.

27. King NA, Hopkins M, Caudwell P, Stubbs RJ, Blundell JE. Individual variability following 12 weeks of supervised exercise: identification and characterisation of compensation for exercise-induced weight loss. Int J Obes. 2008; 32(1): 177-184. 
28. King NA, Lluch A, Stubbs RJ, Blundell JE. High dose exercise does not increase hunger or energy intake in free-living males. Eur J Clin Nutr. 1997; 51(7): 478-483.

29. Laan DJ, Leidy HJ, Lim E, Campbell WW. Effects and reproducibility of aerobic and resistance exercise on appetite and energy intake in young, physically active adults. Appl Physiol Nutr Metab. 2010; 35(6): 842-847.

30. Martins C, Morgan LM, Bloom SR, Robertson MD. Effects of exercise on gut peptides, energy intake and appetite. J Endocrinol. 2007; 193(2): 251-258.

33. Schubert MM, Desbrow B, Sabapathy S, Leveritt M. Acute exercise and energy intake. A meta-analysis. Appetite. 2013; 63: 92-104.

32. Raben A, Tagliabue A, Astrup A. The reproducibility of subjective appetite scores. $\mathrm{Br}$ J Nutr. 1995; 73(4): 517-530.

34. Schubert MM, Sabapathy S, Leveritt M, Desbrow B. Acute exercise and hormones related to appetite regulation: a meta-analysis. Sports Med. 2014; 44(3): 387-403.

35. Siri WE. The gross composition of the body. Adv Biol Med Phys. 1956;4:239-280. 
36. Stubbs RJ, Sepp A, Hughes DA, et al. The effect of graded levels of exercise on energy intake and balance in free-living men consuming their normal diet. Eur J Clin Nutr. 2002; 56(2): 129-140.

37. Ueda S, Yoshikawa T, Katsura Y, Usui T, Fujimoto S. Comparable effects of moderate intensity exercise on changes in anorectic gut hormone levels and energy intake to high intensity exercise. J Endocrinol. 2009; 203(3): 257-364.

38. Unick JL, O’Leary KC, Dorfman L, Thomas JG, Strojacker K, Wing RR. Consistency in compensatory eating responses following acute exercise in inactive, overweight and obese women. Br J Nutr. 2015; 113(7): 1170-1177.

39. Whybrow S, Hughes DA, Ritz P, et al. The effect of an incremental increase in exercise on appetite, eating behaviour and energy balance in lean men and women feeding ad libitum. Br J Nutr. 2008; 100(5): 1109-1115.

40. Wren AM, Seal LJ, Cohen MA et al. Ghrelin enhances appetite and increases food intake in humans. J Clin Endocrinol Metab. 2001; 86(12): 5992-5995.

\section{FIGURE LEGENDS}

Figure 1: mean hunger ratings (exercise minus control) obtained during (a, $n=178$ ) and after exercise (b, $n=118$ ). Values above zero indicate increased hunger during or after exercise; 
values below zero indicate reduced hunger. Horizontal lines represent zones of natural variation across $1 \mathrm{~h}(1 \mathrm{a}: \pm 30 \mathrm{~mm})$ and $2.5 \mathrm{~h}(1 \mathrm{~b}: \pm 20 \mathrm{~mm})$.

Figure 2: Energy intake (exercise minus control) at $(a, n=60)$ one meal consumed within 60 min post-exercise and (b, $n=128)$ at multiple meals after exercise. Each individual data point represents the response for a single study participant. Values above zero indicate increased energy intake after exercise; values below zero indicate reduced energy intake after exercise. Horizontal lines represent zones of natural variation (2a $\pm 1937 \mathrm{~kJ}$; $2 \mathrm{~b} \pm 2138 \mathrm{~kJ}$ ).

Figure 3: circulating acylated ghrelin concentrations (exercise minus control) during (a, $n=$ 118) and over several hours after (b, $n=89$ ) exercise. Each individual data point represents the response for a single study participant. Values above zero indicate increased acylated ghrelin after exercise; values below zero indicate reduced acylated ghrelin after exercise. Horizontal lines represent zones of natural variation (3a $\pm 17.2 \%$; $3 \mathrm{~b} \pm 14.4 \%$ ).

(1)

1

2

\section{SUPPLEMENTAL DIGITAL CONTENT}

SDC 1 (.doc file): studies included in the fasting hunger analyses $(n=192)$

SDC 2 (.doc file): Studies included in the analysis examining hunger responses during exercise $(n=178)$ 
784 SDC 3 (.doc file): Studies included in the analysis examining hunger responses after exercise $785 \quad(\mathrm{n}=118)$

SDC 4 (.doc file): Studies included in energy intake analysis at the first post-exercise meal (n $788=60)$

SDC 5 (.doc file): Studies included in energy intake analysis for all meals after exercise $(\mathrm{n}=$ 791 128)

792 SDC 6 (.doc file): Studies included in fasting acylated ghrelin analysis $(\mathrm{n}=138)$ during exercise $(n=118)$

SDC 8 (.doc file): Studies included in the analysis examining acylated ghrelin responses after exercise $(n=89)$ 